\title{
Comparison of the diagnostic performance of magnetic resonance elastography and Wisteria foribunda agglutinin-positive Mac-2-binding protein in the determination of advanced liver fibrosis stages in patients with chronic liver disease
}

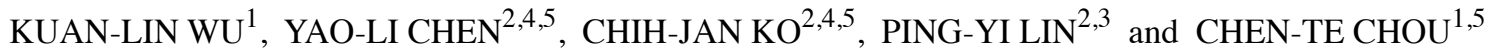 \\ ${ }^{1}$ Department of Diagnostic Radiology, Changhua Christian Hospital; ${ }^{2}$ Transplant Medicine and Surgery Research Center, \\ Changhua Christian Hospital, Changhua 500209; ${ }^{3}$ Department of Nursing, Da-Yeh University, Changhua 515006; \\ ${ }^{4}$ Department of Surgery, Changhua Christian Hospital, Changhua 500209; ${ }^{5}$ School of Medicine, \\ Kaohsiung Medical University, Kaohsiung 807378, Taiwan, R.O.C.
}

Received November 26, 2019; Accepted April 1, 2020

DOI: $10.3892 /$ etm.2020.8922

\begin{abstract}
The present study aimed to compare the accuracy of Wisteria floribunda agglutinin-positive Mac-2-binding protein $\left(\mathrm{WFA}^{+}-\mathrm{M} 2 \mathrm{BP}\right)$ and magnetic resonance elastography (MRE) in determining the liver fibrosis stage in patients with chronic liver disease. A retrospective review of a prospectively maintained database was performed. The eligible patients had hepatic tumors and chronic liver disease, including hepatitis B (HBV) and HCV. All patients underwent blood sampling, MRE and hepatectomy at Changhua Christian Hospital (Changhua, Taiwan). Surgical specimens were used to determine definitive histopathological diagnoses and liver fibrosis stages. Measurement of liver stiffness was performed via MRI. The value of $\mathrm{WFA}^{+}-\mathrm{M} 2 \mathrm{BP}$ in each patient was also assessed. The area under the receiver operating characteristic (ROC) curve (AUC) was measured to compare the diagnostic accuracy of the two examinations. The results indicated that the serum $\mathrm{WFA}^{+}-\mathrm{M} 2 \mathrm{BP}$ levels were able to detect severe liver fibrosis $(\geq F$ 3) in patients with chronic liver disease and performed as well as MRE in patients with HCV. Of the 238 patients enrolled in the present study, 135 had chronic HBV 75 had chronic HCV, 92 had early liver fibrosis (F1-F2) and 139 patients had advanced liver fibrosis (F3-F4). In predicting fibrosis stages $\geq F 3$, MRE had an AUC of 0.89 with a cutoff value of 3.76 and serum $\mathrm{WFA}^{+}-\mathrm{M} 2 \mathrm{BP}$ had an AUC of 0.65 with a cutoff value of 1.32. MRE had higher AUCs
\end{abstract}

Correspondence to: Dr Chen-Te Chou, Department of Diagnostic Radiology, Changhua Christian Hospital, 135 Nan-Hsiao Street, Changhua 500209, Taiwan, R.O.C.

E-mail: 96888@cch.org.tw

Key words: magnetic resonance elastography, hepatic fibrosis, chronic liver disease, Mac-2-binding protein, Wisteria floribunda agglutinin-positive Mac-2-binding protein than serum $\mathrm{WFA}^{+}-\mathrm{M} 2 \mathrm{BP}$ for predicting the severity based on the fibrosis stage in the total cohort and the HBV subgroup. In patients with $\mathrm{HCV}$, no significant differences in diagnostic performance were identified between MRE and serum $\mathrm{WFA}^{+}-\mathrm{M} 2 \mathrm{BP}$. In conclusion, determination of $\mathrm{WFA}^{+}-\mathrm{M} 2 \mathrm{BP}$ as a biomarker for predicting severe liver fibrosis $(\geq F 3)$ is a reliable and non-invasive method and performs as well as MRE in patients with chronic liver disease, particularly those with $\mathrm{HCV}$.

\section{Introduction}

Liver fibrosis is a major consequence of chronic liver disease and its different stages determine the disease prognosis. Progression of fibrosis is linked to portal hypertension, hepatic failure and risk of hepatocellular carcinoma (1). Early treatment and prevention of the progression to liver cirrhosis is important, as the fibrotic process is dynamic and there is a possibility of reversibility (2).

Liver biopsy is considered the gold standard for the evaluation of fibrosis and determination of the stage. However, it is invasive and has major limitations, including sampling variability and interobserver variability (3). There are certain non-invasive methods, including magnetic resonance elastography (MRE) (4), ultrasound-based acoustic radiation force impulse (ARFI) (5) and measurement of biomarkers of fibrosis in serum. MRE is a widely used shear wave imaging technique for the staging of liver fibrosis with high diagnostic accuracy (4). Recently, a novel marker, Wisteria floribunda agglutinin-positive Mac-2-binding protein (WFA ${ }^{+}-\mathrm{M} 2 \mathrm{BP}$; also called M2BPGi), was introduced for determining liver fibrosis and has been confirmed to be a reliable marker for the staging of liver fibrosis (6). To the best of our knowledge, no previous studies have compared the diagnostic accuracy of MRE with that of $\mathrm{WFA}^{+}-\mathrm{M} 2 \mathrm{BP}$. Therefore, the present study aimed to determine serum $\mathrm{WFA}^{+}-\mathrm{M} 2 \mathrm{BP}$ cutoff values for assessing the fibrosis stage and compared the diagnostic accuracy of MRE and $\mathrm{WFA}^{+}-\mathrm{M} 2 \mathrm{BP}$. 


\section{Materials and methods}

Patients. The present study was a retrospective cross-sectional study. Ethical approval was obtained from the Institutional Review Board of Changhua Christian Hospital (Changhua, China; no. 120611). Between April 2015 and April 2017, 251 patients with hepatic tumors underwent hepatectomy at Changhua Christian Hospital (Changhua, Taiwan) and had underlying chronic liver disease. These patients had also received MRE and blood sampling for analysis of serum $\mathrm{WFA}^{+}$-M2BP levels. The time interval between hepatectomy and blood sampling was $<1$ week. Patients who were unable to complete an MRE and those whose MRE was of poor quality were excluded. Finally, 238 patients were enrolled (Fig. 1). Of these 238 patients, 99 had early-stage hepatic fibrosis $(<\mathrm{F} 3)$ and 139 had advanced fibrosis $(\geq F 3)$. Patient characteristics, including age, sex, body mass index (BMI), underlying liver disease and laboratory data were recorded.

MRE. MRI was performed on a 1.5 Tesla magnet system (Aera; Siemens AG) using a 16-channel phased-array body coil for the acquisition of routine clinical MR and MRE images. When paired with an acoustic driver system (Resoundant), the MRE system is capable of generating acoustic shear waves in human livers. A $19-\mathrm{cm}$ diameter, $1.5-\mathrm{cm}$ thick cylindrical passive driver was connected with a flexible plastic tube to an acoustic active driver. The passive driver was placed against the right chest wall located at the level of the xiphoid process. Propagating shear waves were produced from continuous acoustic vibrations at $60 \mathrm{~Hz}$ that were transmitted from the active driver in the liver and they were imaged with an axial 2-dimensional gradient-echo sequence. The parameters of the MRE sequence are described as follows: Repetition time/echo time, 50/22.7; flip angle, $25^{\circ}$; bandwidth, $260 \mathrm{~Hz} /$ pixel; hydrogen resonance frequency, $63.5 \mathrm{MHz}$; acquisition matrix, 256x64; section thickness, $5 \mathrm{~mm}$; and field of view, 400x400 mm. The scanning time of each axial slice was $21 \mathrm{sec}$ per breath-hold. Patients were requested to hold their breath at the end-expiratory stage to obtain a consistent position of the liver for each phase offset. A total of five slices of axial images were acquired for each patient. After all post-processing steps were applied automatically, liver stiffness measurements were determined in kilopascals. If a reflective wave, disturbed wave or artifact occurred on wave imaging, the passive driver was re-positioned on the chest wall to acquire well-propagating wave images. The following elastograms were reviewed automatically by the intrinsic software for artifacts, including significant wave interference and oblique wave propagation. Confidence mapping, which provided regions that had adequate wave amplitudes, was automatically performed by the MRE software.

MRE analysis. All analyses were performed on a dual-screen diagnostic workstation (GE Healthcare). Two abdominal radiologists (CTC and KLW) evaluated the MRE images. One radiologist had $>20$ years of clinical experience in abdominal imaging and the other had three years of experience. The MRE images to be evaluated included the anatomic image sets, the wave image sets and the elastogram sets. The two radiologists were blinded to the patients' clinical information, serum $\mathrm{WFA}^{+}$-M2BP levels and histopathological results. Representative images are displayed in Fig. 2. Fig. 2A provides liver anatomic information from an MRE slice. Fig. 2B is a wave image revealing the pattern of a propagating wave. The two preliminary steps of measurement of liver stiffness were as follows: First, wave images were evaluated for adequate wave quality. Poor propagating imaging was not applied if there was a presence of a reflective wave, disturbing wave or artifact. Furthermore, the areas of the liver with poorly propagating waves were not included. Using the software of the MR unit (MAGNETOM Aera, Skyra and Avantofit, VE11; Siemens Healthineers), elastograms of the MRE slices were generated (Fig. 2C). The elastograms were developed automatically by the intrinsic software to produce the confidence maps (Fig. 2D); the areas without black spots indicate the reliability of the liver stiffness measurement at each voxel. Therefore, the region of interest was manually drawn to include only the parenchyma of the liver on the confidence maps (Fig. 2E). Artifacts including the liver border, hepatic tumors, wave interference and large blood vessels were avoided. The mean stiffness value (in $\mathrm{kPa}$ ) for each elastography image (five slices per patient) was recorded. The overall mean stiffness value of the liver parenchyma was calculated by averaging the mean stiffness values of the five slices for each patient.

Histopathologic analysis. For each patient, five different sections of non-tumoral tissue from the resected specimen were used for histologic examination. The size of histopathologic specimens was determined as the largest tumor diameter plus a free margin of at least $1 \mathrm{~cm}$. All surgical specimens were analyzed by one pathologist with $>10$ years of clinical experience in interpreting hepatic histology. The pathologist was blinded to the MRE results and serum $\mathrm{WFA}^{+}$-M2BP levels, as well as the clinical data. After the tissue was sampled, it was fixed in $10 \%$ neutral buffered formalin in room temperature at $\leq 12 \mathrm{~h}$ and embedded in paraffin. The samples were deparaffinized, and rehydrated usingthrough xylene and a descending ethanol series and subjected to standard techniques of H\&E and Masson trichrome staining. For each patient, the pathologist recorded histologic findings, including the percentage of fat deposition, fibrosis pattern, pattern of necrosis (focal, piecemeal or bridging necrosis) and evidence of portal inflammation. Fibrosis stage (F grade) was assessed using the METAVIR scoring system (7). Fibrosis stage was graded on a scale of 0 to 4 as follows: F0, no fibrosis; F1, mild fibrosis, portal fibrosis without septa; F2, substantial fibrosis, portal fibrosis with few septa; F3, moderate to advanced fibrosis, numerous septa without cirrhosis; and F4, cirrhosis.

Measurement of serum $W_{F A}^{+}-M 2 B P$. Serum samples were collected at the time-point of the MRE measurement. The $\mathrm{WFA}^{+}$-M2BP levels were quantified by a lectin antibody sandwich immunoassay using an automated analyzer (HISCL-800 Sysmex Co.) (8). The $\mathrm{WFA}^{+}-\mathrm{M} 2 \mathrm{BP}$ values conjugated to WFA were indexed with scored values using the following equation $(8,9)$ :

Cutoff index $=\left[\left(\mathrm{WFA}^{+}-\mathrm{M} 2 \mathrm{BP}\right)\right.$ sample- $\left(\mathrm{WFA}^{+}-\mathrm{M} 2 \mathrm{BP}\right)$ $\mathrm{NC}] /\left[\left(\mathrm{WFA}^{+}-\mathrm{M} 2 \mathrm{BP}\right) \mathrm{PC}-\left(\mathrm{WFA}^{+}-\mathrm{M} 2 \mathrm{BP}\right) \mathrm{NC}\right]$ 
Patients with underlying chronic liver disease $(\mathrm{N}=251)$ underwent hepatectomy for primary liver neoplasm between April 2015 and April 2017 and the inclusion

$$
\text { criteria were as follows: }
$$

- Patient had received MRI and MR elastography

- Patient had received blood sampling for $\mathrm{WFA}^{+}-\mathrm{M} 2 \mathrm{BP}$ level

- The time interval between blood sampling and hepatectomy was $\leq 1$ week

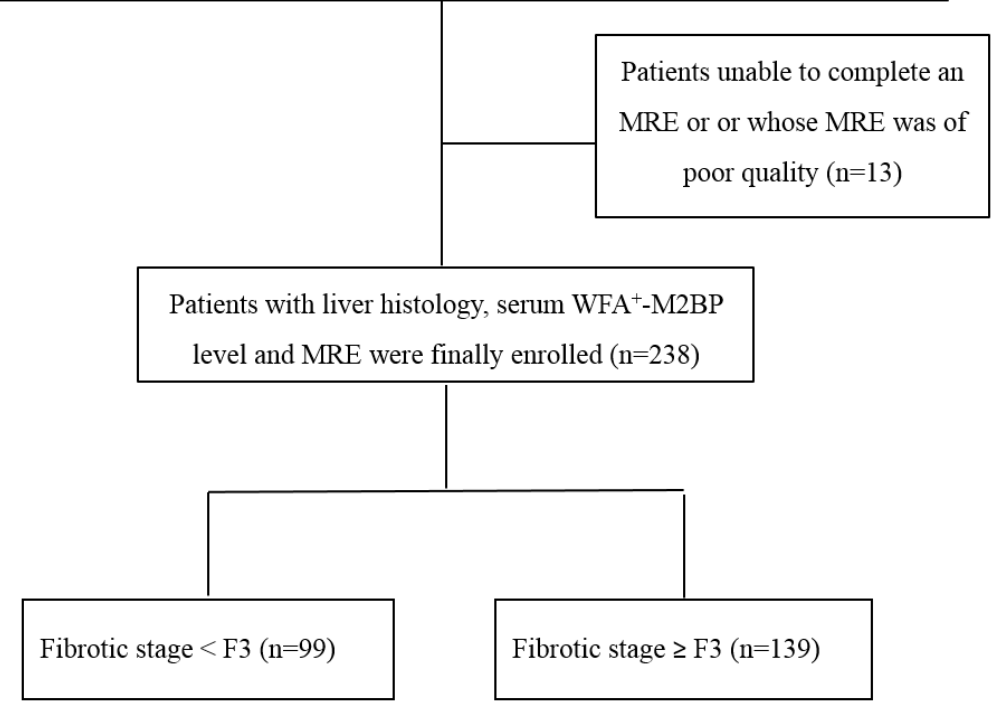

Figure 1. Flow diagram of the patients enrolled in the present study. WFA+-M2BP, Wisteria floribunda agglutinin-positive Mac-2-binding protein; MRE, MR elastography.

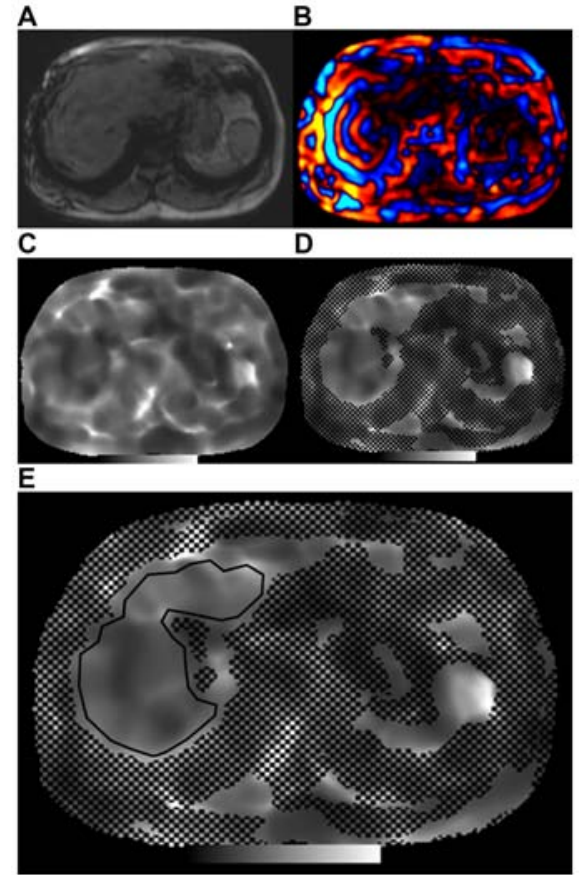

Figure 2. A 43-year-old male with hepatitis $\mathrm{C}$ and hepatocellular carcinoma underwent hepatectomy at an early stage of hepatic fibrosis (fibrosis stage 1). (A) MRE anatomic image revealing the anatomy of the liver. (B) MRE wave image displaying a well-propagating wave through the liver. (C) Processing the images of the shear waves to generate quantitative maps of liver stiffness. (D) Confidence mapping of the elastogram was developed automatically. (E) A region of interest was drawn manually, including the area surrounded by the black line or the area without black spots on the confidence mapping and the value of liver stiffness was $2.67 \mathrm{kPa}$. MRE, MR elastography. where $\left[\mathrm{WFA}^{+}-\mathrm{M} 2 \mathrm{BP}\right]$ sample is the $\mathrm{WFA}^{+}-\mathrm{M} 2 \mathrm{BP}$ level in the serum sample, $\mathrm{PC}$ is the positive control and $\mathrm{NC}$ is the negative control. The positive control was supplied by the manufacturer as a calibration solution preliminarily standardized to yield a cutoff value of 1.0 .

Statistical analyses. Continuous variables, including age and laboratory test results, are expressed as the mean \pm standard deviation. Pearson's correlation coefficient was determined to examine the association between serum $\mathrm{WFA}^{+}$-M2BP levels and the BMI. A receiver operating characteristic (ROC) curve analysis, which was performed by plotting sensitivity against 1-specificity, and the highest Youden index (the highest sensitivity + specificity) were used to determine the optimal cutoff values for MRE average and $\mathrm{WFA}^{+}-\mathrm{M} 2 \mathrm{BP}$ level. The area under the ROC curve (AUC) was determined to evaluate the diagnostic accuracy for different stages of hepatic fibrosis in the total cohort and in subgroups of patients with chronic hepatitis B (HBV) or HCV infection. Using the approach of DeLong et al (10), the statistical significance of the difference between two AUCs was evaluated. $\mathrm{P}<0.05$ was considered to indicate statistical significance. All statistical analyses were performed with MedCalc for Windows, version 16.8.4 (MedCalc Software bvba).

\section{Results}

Patient characteristics. A total of 238 patients were enrolled in the present study. The clinical characteristics of the 
patients are presented in Table I. The mean age of the patients with HCC patients was $61.66 \pm 11.12$ years (193 males and 45 females). According to the Metavir scores (F0-F4), 7 had no evidence of fibrosis (F0), 32 had mild fibrosis (F1), 60 presented with substantial fibrosis (F2), 55 had advanced fibrosis without evidence of cirrhosis (F3) and $84 \mathrm{had}$ cirrhosis (F4). Among the enrolled patients, 135 had chronic $\mathrm{HBV}, 75$ had HCV, 12 had combined chronic HBV and HCV, 13 had alcoholic liver disease and 3 had cryptogenic causes resulting in liver fibrosis. The mean BMI was $24.70 \pm 3.53$. The serum $\mathrm{WFA}^{+}-\mathrm{M} 2 \mathrm{BP}$ level was not significantly correlated with the BMI $(\mathrm{r}=0.021 ; \mathrm{P}=0.752)$. No steatosis was identified in 121 patients, 99 patients had mild steatosis, moderate steatosis was identified in 14 patients and 4 patients had severe steatosis.

Application of MRE and $W F A^{+}-M 2 B P$ predicting to predict hepatic fibrosis stage. ROC curve analysis was used to estimate the cutoff value, sensitivity and specificity of MRE and serum $\mathrm{WFA}^{+}-\mathrm{M} 2 \mathrm{BP}$ in predicting each fibrosis stage. The results are summarized in Tables II and III, respectively. With a liver stiffness cutoff value of $3.762 \mathrm{kPa}$, MRE yielded a sensitivity of $66.91 \%$, a specificity of $95.96 \%$ and an AUC of 0.886 for predicting $\geq \mathrm{F} 2$ in the total cohort of 238 patients. Its predictive ability was statistically significant $(\mathrm{P}<0.001)$, and similar results were also observed for the prediction of stages $\geq F 3$ and F4. Similar results for estimating each fibrosis stage were also obtained in the HBV and HCV subgroups. With a cutoff value of $1.32, \mathrm{WFA}^{+}-\mathrm{M} 2 \mathrm{BP}$ had a sensitivity of $58.99 \%$, a specificity of $67.68 \%$ and an AUC of 0.649 for predicting stage $\geq F 2$ in all patients, and the predictive ability was statistically significant. Similar results were obtained for predicting stages $\geq F 3$ and F4, even in the HBV and HCV subgroups. However, there was no significant difference between F0-1 and F2-4 in the total cohort and in the HCV subgroup, and there was also no significance for differentiating F0-1 from F2-4 and F0-2 from F3-4 in the HBV subgroup. In Table IV, the HBV subgroup was divided into two groups according to the level of alanine aminotransferase (ALT; $\geq 40 \mathrm{mg} / \mathrm{dl}$ vs. $<40 \mathrm{mg} / \mathrm{dl}$ ). In the HBV subgroup with $\geq 40 \mathrm{mg} / \mathrm{dl}$ of ALT, WFA ${ }^{+}-\mathrm{M} 2 \mathrm{BP}$ was able to significantly to distinguish fibrosis stage $\mathrm{F} 4$ from $\mathrm{F} 0-3$ with a cutoff value of $1.41(\mathrm{P}=0.007)$. Conversely, in the HBV subgroup with $<40 \mathrm{mg} / \mathrm{dl}$ of ALT, WFA+-M2BP was not able to significantly differentiate between patients with each fibrosis stage as F0-3 and those with F4.

Comparison of diagnostic ability between MRE and $W F A^{+}-M 2 B P$. The diagnostic performance of $\mathrm{WFA}^{+}-\mathrm{M} 2 \mathrm{BP}$ to distinguish between fibrosis stages F0-2 and F3-4 by using ROC curve analysis was compared with that of MRE (Table V and Fig. 3). The prediction had statistical significance for the total cohort and the HBV subgroup, and in each case, MRE had a significantly larger AUC than $\mathrm{WFA}^{+}$-M2BP for detecting severe fibrosis $(\geq F 3)$. A similar result was obtained in the HCV subgroup, with a larger AUC for MRE (0.88) than that for $\mathrm{WFA}^{+}-\mathrm{M} 2 \mathrm{BP}$ (0.76), although there was no significant difference.

\section{Discussion}

The present results revealed that serum $\mathrm{WFA}^{+}-\mathrm{M} 2 \mathrm{BP}$ had a better diagnostic performance for determining severe
Table I. Clinical characteristics of the patients enrolled in the present study $(\mathrm{n}=238)$.

\begin{tabular}{|c|c|}
\hline Parameter & Total number of pati \\
\hline Age (years) & $61.66 \pm 11.12$ \\
\hline BMI $\left(\mathrm{kg} / \mathrm{m}^{2}\right)$ & $24.70 \pm 3.53$ \\
\hline \multicolumn{2}{|l|}{ Gender } \\
\hline Male & $193(81.1)$ \\
\hline Female & $45(18.9)$ \\
\hline \multicolumn{2}{|l|}{ Underlying disease } \\
\hline HBV & $135(56.7)$ \\
\hline $\mathrm{HCV}$ & $75(31.5)$ \\
\hline $\mathrm{HBV}+\mathrm{HCV}$ & $12(5.0)$ \\
\hline Alcoholic liver disease & $13(5.5)$ \\
\hline Cryptogenic causes & $3(1.3)$ \\
\hline \multicolumn{2}{|l|}{ METAVIR score (\%) } \\
\hline 0 & $7(2.9)$ \\
\hline 1 & $32(13.4)$ \\
\hline 2 & $60(25.2)$ \\
\hline 3 & $55(23.1)$ \\
\hline 4 & $84(35.3)$ \\
\hline \multicolumn{2}{|l|}{ Hepatic steatosis ${ }^{\mathrm{a}}$} \\
\hline 0 & $121(50.8)$ \\
\hline 1 & $99(41.6)$ \\
\hline 2 & $14(5.9)$ \\
\hline 3 & $4(1.7)$ \\
\hline $\operatorname{AST}(\mathrm{U} / \mathrm{l})$ & $65.34 \pm 67.82$ \\
\hline $\operatorname{ALT}(\mathrm{U} / \mathrm{l})$ & $58.64 \pm 54.31$ \\
\hline Bilirubin (mg/d) & $0.85 \pm 0.40$ \\
\hline $\operatorname{PLT}\left(10^{3} / \mu 1\right)$ & $174.01 \pm 71.25$ \\
\hline APTT (sec) & $32.82 \pm 4.62$ \\
\hline PT (sec) & $11.41 \pm 1.09$ \\
\hline INR & $1.10 \pm 0.59$ \\
\hline \multicolumn{2}{|c|}{ Necroinflammatory activity } \\
\hline No & $83(34.9)$ \\
\hline Yes & $155(65.1)$ \\
\hline WFA+-M2BP (C.O.I.) & $1.74 \pm 1.58$ \\
\hline MRE average (kPa) & $3.82 \pm 1.22$ \\
\hline
\end{tabular}

${ }^{a}$ Ultrasonographic fatty liver grades. Values are expressed as the mean \pm standard deviation or $\mathrm{n}(\%)$. HBV, hepatitis B virus; $\mathrm{HCV}$, hepatitis C virus; AST, aspartate aminotransferase; ALT, alanine aminotransferase; MRE, MR elastography; WFA+-M2BP, Wisteria floribunda agglutinin-positive Mac-2-binding protein; BMI, body mass index; PLT, platelets; APTT, activated partial thromboplastin time; PT, prothrombin time; INR, international normalized ratio.

hepatic fibrosis $(\geq F 3)$ in patients with $\mathrm{HCV}$ infection (AUC, 0.76) than in patients with chronic HBV infection (AUC, 0.59). Among patients with HBV infection, there was no statistically significant difference except for in the subgroup of advanced hepatic fibrosis stage $(\geq F 4)$. Similar results were obtained for MRE (AUC, 0.88 for $\mathrm{HCV}$ ) and there was no 
Table II. Diagnostic performance of MR elastography in the estimation of the liver fibrosis stage using receiver operating characteristic analysis.

\begin{tabular}{lccccrr}
\hline Cohort/comparison & Cut-off value & AUC & $95 \%$ CI & Sensitivity & Specificity & P-value \\
\hline Total (n=238) & & & & & & \\
F0-F1 vs. F2-F4 & 3.158 & 0.898 & $0.853-0.934$ & 77.39 & 89.74 & $<0.001$ \\
F0-F2 vs. F3-F4 & 3.762 & 0.886 & $0.839-0.923$ & 66.91 & 95.96 & $<0.001$ \\
F0-F3 vs. F4 & 3.983 & 0.904 & $0.859-0.938$ & 85.71 & 88.31 & $<0.001$ \\
HBV (n=135) & & & & & & \\
F0-F1 vs. F2-F4 & 2.768 & 0.879 & $0.812-0.929$ & 93.22 & 76.47 & $<0.001$ \\
F0-F2 vs. F3-F4 & 3.668 & 0.862 & $0.792-0.915$ & 63.75 & 94.55 & $<0.001$ \\
F0-F3 vs. F4 & 3.893 & 0.843 & $0.770-0.900$ & 75.51 & 87.21 & $<0.001$ \\
HCV (n=75) & & & & & & \\
F0-F1 vs. F2-F4 & 3.432 & 0.912 & $0.823-0.965$ & 72.31 & 100.00 & $<0.001$ \\
F0-F2 vs. F3-F4 & 3.768 & 0.879 & $0.783-0.943$ & 69.39 & 96.15 & $<0.001$ \\
F0-F3 vs. F4 & 3.900 & 0.975 & $0.909-0.997$ & 100.00 & 87.50 & $<0.001$ \\
\hline
\end{tabular}

AUC, area under the receiver operating characteristic curve.

Table III. Diagnostic performance of serum Wisteria floribunda agglutinin-positive Mac-2-binding protein in the estimation of the liver fibrosis stage using receiver operating characteristic analysis.

\begin{tabular}{lcccccr}
\hline Cohort/comparison & Cut-off value & AUC & $95 \%$ CI & Sensitivity & Specificity & P-value \\
\hline $\begin{array}{l}\text { Total (n=238) } \\
\text { F0-F1 vs. F2-F4 }\end{array}$ & 1.11 & 0.579 & $0.513-0.642$ & 60.30 & 64.10 & 0.088 \\
F0-F2 vs. F3-F4 & 1.32 & 0.649 & $0.585-0.710$ & 58.99 & 67.68 & $<0.001$ \\
F0-F3 vs. F4 & 1.32 & 0.657 & $0.592-0.717$ & 64.29 & 61.04 & $<0.001$ \\
HBV (n=135) & & & & & & \\
F0-F1 vs. F2-F4 & 1.11 & 0.566 & $0.478-0.651$ & 52.54 & 76.47 & 0.346 \\
F0-F2 vs. F3-F4 & 1.19 & 0.593 & $0.506-0.677$ & 55.00 & 67.27 & 0.060 \\
F0-F3 vs. F4 & 1.07 & 0.617 & $0.529-0.699$ & 65.31 & 55.81 & 0.023 \\
HCV (n=75) & & & & & & \\
F0-F1 vs. F2-F4 & 2.21 & 0.622 & $0.503-0.732$ & 46.15 & 90.00 & 0.143 \\
F0-F2 vs. F3-F4 & 2.21 & 0.755 & $0.642-0.847$ & 59.18 & 92.31 & $<0.001$ \\
F0-F3 vs. F4 & 2.45 & 0.728 & $0.613-0.825$ & 59.26 & 79.17 & 0.001 \\
\hline
\end{tabular}

AUC, area under the receiver operating characteristic curve.

statistically significant difference from the diagnostic value of $\mathrm{WFA}^{+}-\mathrm{M} 2 \mathrm{BP}(\mathrm{P}=0.073)$. However, in the entire study group and the HBV subgroup, the diagnostic performance of $\mathrm{WFA}^{+}-\mathrm{M} 2 \mathrm{BP}$ in detecting severe fibrosis was significantly lower than that of MRE $(\mathrm{P}<0.001)$. Kuno et al (9) first described the use of serum $\mathrm{WFA}^{+}-\mathrm{M} 2 \mathrm{BP}$ as a non-invasive biomarker for hepatic fibrosis and it now serves as a reliable serum biomarker. Toshima et al (11) reported that the AUC value for using $\mathrm{WFA}^{+}-\mathrm{M} 2 \mathrm{BP}$ to predict the hepatic fibrosis stage was similar to that for ARFI and the diagnostic performance of $\mathrm{WFA}^{+}-\mathrm{M} 2 \mathrm{BP}$ is superior to that of other surrogate markers, including the aspartate aminotransferase-to-platelet ratio index. $\mathrm{WFA}^{+}-\mathrm{M} 2 \mathrm{BP}$ is also useful for evaluating liver fibrosis in patients with non-alcoholic fatty liver disease (12), autoimmune hepatitis (13), primary biliary cirrhosis (14) and biliary atresia (15). The present study also demonstrated that serum $\mathrm{WFA}^{+}-\mathrm{M} 2 \mathrm{BP}$ had satisfactory results in determining severe hepatic fibrosis $(\geq \mathrm{F} 3)$ in patients with chronic liver disease (AUC, 0.65) and even in the HCV subgroup (AUC, 0.76).

In the present study, it was determined that the AUC for $\mathrm{WFA}^{+}-\mathrm{M} 2 \mathrm{BP}$ serum levels in the prediction of fibrosis stages $\geq \mathrm{F} 3$ was 0.65 in the total cohort, 0.59 in the HBV subgroup and 0.76 in the HCV subgroup. These AUC values were similar to the results of prior studies only for patients with $\mathrm{HCV}$ infection and the AUCs for predicting patients with chronic HBV infection were lower than those in the previous studies. Toshima et al (11) determined that the stage of hepatic 
Table IV. Serum $\mathrm{WFA}^{+}$-M2BP performance in estimating the liver fibrosis stage among patients with hepatitis B virus infection $(\mathrm{n}=135)$ using ROC analysis.

\begin{tabular}{|c|c|c|c|c|c|c|}
\hline Cohort/comparison & Cut-off value & AUC & $95 \% \mathrm{CI}$ & Sensitivity & Specificity & P-value \\
\hline \multicolumn{7}{|l|}{ ALT $\geq 40(n=63)$} \\
\hline F0-F1 vs. F2-F4 & 1.11 & 65.8 & $0.528-0.773$ & 66.07 & 71.43 & 0.182 \\
\hline F0-F2 vs. F3-F4 & 1.32 & 63.4 & $0.503-0.752$ & 62.50 & 69.57 & 0.067 \\
\hline F0-F3 vs. F4 & 1.41 & 68.9 & $0.560-0.799$ & 62.96 & 75.00 & 0.007 \\
\hline \multicolumn{7}{|l|}{ ALT $<40(n=72)$} \\
\hline F0-F1 vs. F2-F4 & 1.02 & 52.1 & $0.400-0.640$ & 56.45 & 20.00 & 0.816 \\
\hline F0-F2 vs. F3-F4 & 0.91 & 56.1 & $0.439-0.677$ & 55.00 & 65.62 & 0.381 \\
\hline F0-F3 vs. F4 & 0.98 & 53.5 & $0.414-0.653$ & 54.55 & 62.00 & 0.641 \\
\hline
\end{tabular}

AUC, area under the receiver operating characteristic curve; ALT, alanine aminotransferase.

Table V. Comparison of the diagnostic performance MRE and WFA+-M2BP in the prediction of liver fibrosis stage $\geq F 3$ (F0-F2 vs. F3-F4) using ROC analysis.

\begin{tabular}{|c|c|c|c|c|c|c|c|c|}
\hline Cohort/modality & $\begin{array}{l}\text { Cut-off } \\
\text { value }\end{array}$ & AUC & $95 \% \mathrm{CI}$ & Sensitivity & Specificity & $\mathrm{P}$-value & $\begin{array}{c}\text { Difference between } \\
\text { areas (MRE vs. Mac 2) }\end{array}$ & P-value \\
\hline Total $(n=238)$ & & & & & & & 0.237 & $<0.001$ \\
\hline MRE & 3.76 & 0.886 & $0.839-0.923$ & 66.91 & 95.96 & $<0.001$ & & \\
\hline $\mathrm{WFA}^{+}-\mathrm{M} 2 \mathrm{BP}$ & 1.32 & 0.649 & $0.585-0.710$ & 58.99 & 67.68 & $<0.001$ & & \\
\hline $\operatorname{HBV}(n=135)$ & & & & & & & 0.268 & $<0.001$ \\
\hline MRE & 3.67 & 0.862 & $0.792-0.915$ & 63.75 & 94.55 & $<0.001$ & & \\
\hline $\mathrm{WFA}^{+}-\mathrm{M} 2 \mathrm{BP}$ & 1.19 & 0.593 & $0.506-0.677$ & 55.00 & 67.27 & 0.060 & & \\
\hline $\mathrm{HCV}(\mathrm{n}=75)$ & & & & & & & 0.124 & 0.073 \\
\hline MRE & 3.77 & 0.879 & $0.783-0.943$ & 69.39 & 96.15 & $<0.001$ & & \\
\hline $\mathrm{WFA}^{+}-\mathrm{M} 2 \mathrm{BP}$ & 2.21 & 0.755 & $0.642-0.847$ & 59.18 & 92.31 & $<0.001$ & & \\
\hline
\end{tabular}

AUC, area under the receiver operating characteristic curve; MRE, MR elastography; WFA+-M2BP, Wisteria floribunda agglutinin-positive Mac-2-binding protein.
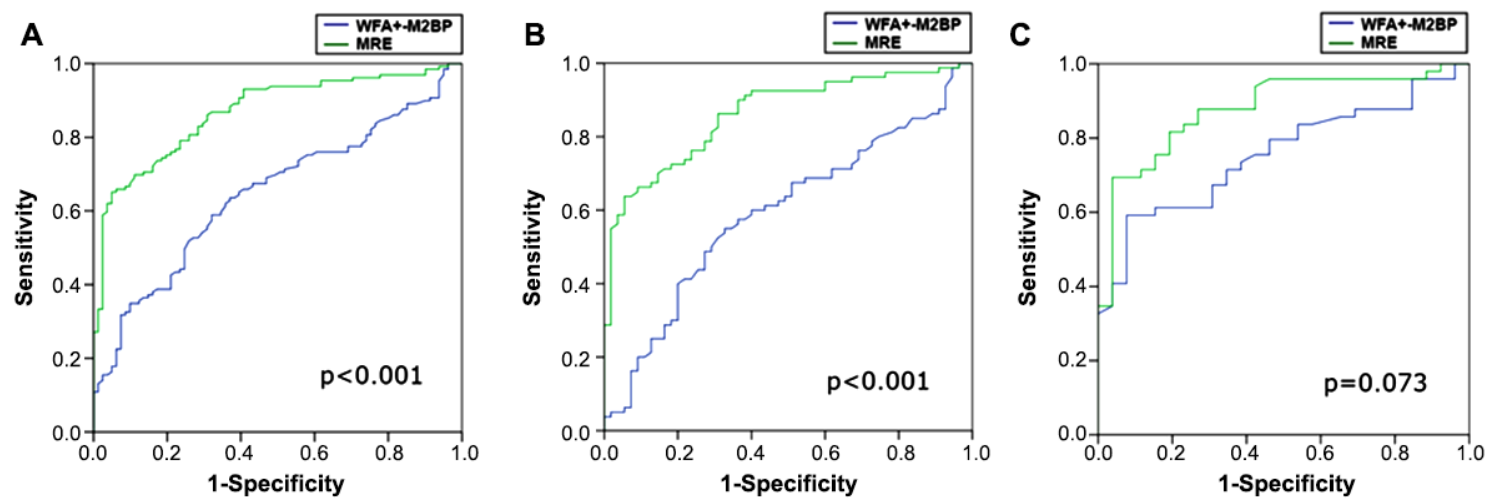

Figure 3. Comparison of the diagnostic performance MRE and WFA+-M2BP in the identification of liver fibrosis stage $\geq \mathrm{F} 3$ using receiver operating characteristic analysis based on a calculation of the area under the ROC curve. (A) All patients. (B) Patients with chronic HBV infection. (C) Patients with chronic $\mathrm{HCV}$ infection. MRE, MR elastography; WFA+-M2BP, Wisteria floribunda agglutinin-positive Mac-2-binding protein.

fibrosis may be accurately estimated using the serum levels of $\mathrm{WFA}^{+}-\mathrm{M} 2 \mathrm{BP}$, with an AUC of 0.81 in determining an advanced histologic stage $(\geq \mathrm{F} 3)$, an AUC of 0.80 in the HCV subgroup and an AUC of 0.62 in the HBV subgroup. Their study included only a few patients with fibrosis stage $\mathrm{F} 3(\mathrm{n}=16)$ and HBV infection $(n=21)$. Nishikawa et al $(16)$ reported an AUC 
of 0.72 for determining severe fibrosis in the HBV subgroup (111 patients) and an AUC of 0.83 in the HCV subgroup (275 patients). The reasons for the discrepancy remain elusive, but they may be due to different patient numbers in the HBV and HCV subgroups, as well as differences in viral activity and fibrosis period. Further validation will be required in future studies. Furthermore, percutaneous liver needle biopsy performed for assessing the hepatic fibrosis stage in the study by Nishikawa et al (16) may have been associated with sampling errors, although numerous patients with HBV and $\mathrm{HCV}$ were enrolled. However, the present results suggest that the diagnostic performance of serum $\mathrm{WFA}^{+}-\mathrm{M} 2 \mathrm{BP}$ is more accurate in $\mathrm{HCV}$ patients than in $\mathrm{HBV}$ patients and caution should be taken when using $\mathrm{WFA}^{+}-\mathrm{M} 2 \mathrm{BP}$ to assess the degree of liver fibrosis in patients with HBV.

In the present study, the AUC value for serum $\mathrm{WFA}^{+}-\mathrm{M} 2 \mathrm{BP}$ was higher in patients with $\mathrm{HCV}$ infection than in patients with HBV infection and the same trend was observed for MRE. However, the serum $\mathrm{WFA}^{+}-\mathrm{M} 2 \mathrm{BP}$ value demonstrated a better diagnostic performance in distinguishing between fibrosis stages F0-3 and F4 in patients with $\mathrm{HBV}$ and $\geq 40 \mathrm{mg} / \mathrm{dl}$ of ALT than in patients with HBV and $<40 \mathrm{mg} / \mathrm{dl}$ of ALT. ALT levels are important to characterize the phase of infection (17). A higher ALT level is indicative of a more severe inflammatory condition that may contribute to more advanced fibrosis and is associated with a better diagnostic accuracy of serum $\mathrm{WFA}^{+}$-M2BP.

Nishikawa et al (16) observed a similar phenomenon and reported higher cut-off points of $\mathrm{WFA}^{+}-\mathrm{M} 2 \mathrm{BP}$ levels for identifying $F 4, \geq F 3$ and $\geq F 2$ in their $\mathrm{HCV}$ subgroup compared to their HBV subgroup, with a significant difference even for the same degree of liver fibrosis. This may be due to different patterns of liver fibrosis between $\mathrm{HBV}$ and $\mathrm{HCV}$, as the presence of thicker fibrotic septa in HCV results in a larger amount of fibrotic tissue (18) and in patients with hepatitis $\mathrm{C}$ infection, it may also be faster progression of hepatic lesions compared with in patients with hepatitis $\mathrm{B}$, which is a result of continuous viral replication, inflammation and fibrogenesis after disease progression to cirrhosis (18). Conversely, inflammation was reported to become inactive in most cases with advanced hepatic fibrosis, as $\mathrm{HBe}$ seroconversion leads to termination of the progression of liver fibrosis $(19,20)$. Further studies are required to evaluate the individual cutoff values in other etiologies of chronic liver disease.

Blood sampling to obtain serum $\mathrm{WFA}^{+}-\mathrm{M} 2 \mathrm{BP}$ levels is a convenient and non-invasive method for evaluating advanced hepatic fibrosis in patients with chronic liver disease. Based on the present study, the diagnostic ability is similar to that of MRE, particularly in patients with chronic hepatitis. In contrast to MR, the assessment time is short and claustrophobia is not an issue. Furthermore, owing to its simple and reproducible characteristics, MRE may be applied to period surveillance under antiviral therapy of chronic hepatitis. The American Association for the Study of Liver Diseases guidelines also concluded that the patients at the highest risk of liver-associated complications, including $\mathrm{HCV}$ with advanced hepatic fibrosis, benefit from treatment (21). Therefore, serum $\mathrm{WFA}^{+}$-M2BP levels may be used for periodic surveillance of patients with advanced liver disease who are not able to receive treatment immediately.
Of note, the present study had several limitations. It was a retrospective study and there was low statistical power due to the different case numbers of each etiology of chronic liver disease. In particular, the HBV and HCV subgroups had 135 and 75 patients, respectively. A prospective study may be required for further clarification. In addition, the patients of the present study were diagnosed with liver tumors and received hepatectomy. The hepatic fibrosis stage was determined based on the normal peritumoral liver tissue in the surgical specimen; assessment of the whole liver fibrosis stage may be performed in a future study.

In conclusion, the diagnostic accuracy of serum $\mathrm{WFA}^{+}-\mathrm{M} 2 \mathrm{BP}$ is reliable and comparable to that of MRE in determining severe hepatic fibrosis associated with chronic liver disease. Owing to its rapid, simple and non-invasive measurement, serum $\mathrm{WFA}^{+}-\mathrm{M} 2 \mathrm{BP}$ may be useful in the initial screening and therapeutic follow-up for patients with chronic liver disease and most suitable for the patient with hepatitis $\mathrm{C}$ infection.

\section{Acknowledgements}

The authors would like to express gratitude to the pathologist (Dr. Hui-Ting Hsu, Department of Surgical Pathology, Changhua Christian Hospital, Changhua, Taiwan) for her assistance with the interpretation of the hepatic histology data.

\section{Funding}

No funding was received.

\section{Availability of data and materials}

The datasets used and/or analyzed during the current study are available from the corresponding author on reasonable request.

\section{Authors' contributions}

KLW, YLC, CJK, PYL and CTC contributed to the study conception. KLW, YLC and CTC drafted the manuscript. CJK, PYL and CTC contributed to the study design, analysis and interpretation of data. KLW, YLC and CTC critically revised the manuscript for important intellectual content. All authors read and approved the final manuscript.

\section{Ethics approval and consent to participate}

All procedures followed were in accordance with the ethical standards of the responsible committee on human experimentation (Changhua Christian Hospital, Changhua, Taiwan) and with the Helsinki Declaration of 1964 and later versions. Institutional Review Board approval was obtained from Changhua Christian Hospital (Changhua, Taiwan; approval dated 20 May 2016).

\section{Patient consent for publication}

Not applicable. 


\section{Competing interests}

The authors declare that they have no competing interests.

\section{References}

1. Barr RG, Ferraioli G, Palmeri ML, Goodman ZD, Garcia-Tsao G, Rubin J, Garra B, Myers RP, Wilson SR, Rubens D and Levine D: Elastography assessment of liver fibrosis: Society of radiologists in ultrasound consensus conference statement. Radiology 276 845-861, 2015.

2. Marcellin P, Gane E, Buti M, Afdhal N, Sievert W, Jacobson IM, Washington MK, Germanidis G, Flaherty JF, Aguilar Schall R, et al: Regression of cirrhosis during treatment with tenofovir disoproxil fumarate for chronic hepatitis B: A 5-year open-label follow-up study. Lancet 381: 468-475, 2013

3. Bedossa P, Dargère D and Paradis V: Sampling variability of liver fibrosis in chronic hepatitis C. Hepatology 38: 1449-1457, 2003.

4. Cui J, Heba E, Hernandez C, Haufe W, Hooker J, Andre MP, Valasek MA, Aryafar H, Sirlin CB and Loomba R: Magnetic resonance elastography is superior to acoustic radiation force impulse for the diagnosis of fibrosis in patients with biopsy-proven nonalcoholic fatty liver disease: A prospective study. Hepatology 63: 453-461, 2016.

5. Toshima T, Shirabe K, Takeishi K, Motomura T, Mano Y, Uchiyama H, Yoshizumi T, Soejima Y, Taketomi A and Maehara Y: New method for assessing liver fibrosis based on acoustic radiation force impulse: A special reference to the difference between right and left liver. J Gastroenterol 46 : 705-711, 2011.

6. Ito K, Murotani K, Nakade Y, Inoue T, Nakao H, Sumida Y, Kamada Y and Yoneda M: Serum Wisteria floribunda agglutinin-positive Mac-2-binding protein levels and liver fibrosis: A meta-analysis. J Gastroenterol Hepatol 32: 1922-1930, 2017.

7. Bedossa P and Poynard T: An algorithm for the grading of activity in chronic hepatitis C. Hepatology 24: 289-293, 1996.

8. Kuno A, Sato T, Shimazaki H, Unno S, Saitou K, Kiyohara K, Sogabe M, Tsuruno C, Takahama Y, Ikehara Y and Narimatsu H: Reconstruction of a robust glycodiagnostic agent supported by multiple lectin-assisted glycan profiling. Proteomics Clin Appl 7: 642-647, 2013.

9. Kuno A, Ikehara Y, Tanaka Y, Ito K, Matsuda A, Sekiya S, Hige S, Sakamoto M, Kage M, Mizokami M and Narimatsu H: A serum 'sweet-doughnut' protein facilitates fibrosis evaluation and therapy assessment in patients with viral hepatitis. Sci Rep 3: 1065,2013

10. DeLong ER, DeLong DM and Clarke-Pearson DL: Comparing the areas under two or more correlated receiver operating char acteristic curves: A nonparametric approach. Biometrics 44: $837-845,1988$
11. Toshima T, Shirabe K, Ikegami T, Yoshizumi T, Kuno A, Togayachi A, Gotoh M, Narimatsu H, Korenaga M, Mizokami M, et al: A novel serum marker, glycosylated Wisteria floribunda agglutinin-positive Mac-2 binding protein (WFA(+)-M2BP), for assessing liver fibrosis. J Gastroenterol 50: 76-84, 2015.

12. Abe M, Miyake T, Kuno A, Imai Y, Sawai Y, Hino K, Hara Y, Hige S, Sakamoto M, Yamada G, et al: Association between Wisteria floribunda agglutinin-positive Mac-2 binding protein and the fibrosis stage of non-alcoholic fatty liver disease. J Gastroenterol 50: 776-784, 2015.

13. Nishikawa H, Enomoto H, Iwata $Y$, Hasegawa K, Nakano C, Takata R, Nishimura T, Yoh K, Aizawa N, Sakai Y, et al: Clinical significance of serum Wisteria floribunda agglutinin positive Mac-2-binding protein level and high-sensitivity C-reactive protein concentration in autoimmune hepatitis. Hepatol Res 46: 613-621, 2016.

14. Umemura T, Joshita S, Sekiguchi T, Usami Y, Shibata S, Kimura T, Komatsu M, Matsumoto A, Ota M and Tanaka E: Serum wisteria floribunda agglutinin-positive Mac-2-binding protein level predicts liver fibrosis and prognosis in primary biliary cirrhosis. Am J Gastroenterol 110: 857-864, 2015.

15. Yamada N, Sanada Y, Tashiro M, Hirata Y, Okada N, Ihara Y, Urahashi T and Mizuta K: Serum Mac-2 binding protein glycosylation isomer predicts grade F4 liver fibrosis in patients with biliary atresia. J Gastroenterol 52: 245-252, 2017.

16. Nishikawa H, Enomoto H, Iwata Y, Kishino K, Shimono Y, Hasegawa K, Nakano C, Takata R, Nishimura T, Yoh K, et al: Serum Wisteria floribunda agglutinin-positive Mac-2-binding protein for patients with chronic hepatitis B and C: A comparative study. J Viral Hepat 23: 977-984, 2016.

17. Terrault NA, Bzowej NH, Chang KM, Hwang JP, Jonas MM and Murad MH: AASLD guidelines for treatment of chronic hepatitis B. Hepatology 63: 261-283, 2016.

18. Kage M, Shimamatu K, Nakashima E, Kojiro M, Inoue O and Yano M: Long-term evolution of fibrosis from chronic hepatitis to cirrhosis in patients with hepatitis C: Morphometric analysis of repeated biopsies. Hepatology 25: 1028-1031, 1997.

19. McMahon BJ: The natural history of chronic hepatitis B virus infection. Hepatology 49 (Suppl 5): S45-S55, 2009.

20. Bedossa P: Reversibility of hepatitis B virus cirrhosis after therapy: Who and why? Liver Int 35 (Suppl 1): S78-S81, 2015.

21. AASLD/IDSA HCV Guidance Panel: Hepatitis C guidance: AASLD-IDSA recommendations for testing, managing, and treating adults infected with hepatitis $C$ virus. Hepatology 62 : 932-954, 2015.

This work is licensed under a Creative Commons Attribution-NonCommercial-NoDerivatives 4.0 International (CC BY-NC-ND 4.0) License. 